\title{
Age-related changes in the bimanual advantage and in brain oscillatory activity during tapping movements suggest a decline in processing sensory reafference
}

\author{
Etienne Sallard · Lucas Spierer • Catherine Ludwig • \\ Marie-Pierre Deiber · Jérôme Barral
}

Received: 22 May 2013 / Accepted: 26 October 2013 / Published online: 15 November 2013

(C) Springer-Verlag Berlin Heidelberg 2013

\begin{abstract}
Deficits in the processing of sensory reafferences have been suggested as accounting for age-related decline in motor coordination. Whether sensory reafferences are accurately processed can be assessed based on the bimanual advantage in tapping: because of tapping with an additional hand increases kinesthetic reafferences, bimanual tapping is characterized by a reduced inter-tap interval variability than unimanual tapping. A suppression of the bimanual advantage would thus indicate a deficit in sensory reafference. We tested whether elderly indeed show a reduced bimanual advantage by measuring unimanual (UM) and bimanual (BM) self-paced tapping performance in groups of young $(n=29)$ and old $(n=27)$ healthy adults. Electroencephalogram was recorded to assess the underlying patterns of oscillatory activity, a neurophysiological mechanism advanced to support the integration of sensory reafferences. Behaviorally, there was a significant interaction between the factors tapping condition and age group at the level of the inter-tap interval variability, driven by a lower variability in BM than
\end{abstract}

E. Sallard $(\bowtie) \cdot$ J. Barral

Institute of Sport Sciences (GRISSUL), University of Lausanne, Quartier UNIL-Mouline, Bâtiment Géopolis, 1015 Lausanne, Switzerland

e-mail: Etienne.Sallard@unil.ch

L. Spierer

Neurology Unit, Department of Medicine, Faculty of Sciences, University of Fribourg, 1700 Fribourg, Switzerland

C. Ludwig

Faculty of Psychology and Educational Sciences, Center for the Interdisciplinary Study of Gerontology and Vulnerability, University of Geneva, Geneva, Switzerland

M.-P. Deiber

Faculty of Medicine, INSERM U1039, La Tronche, France
UM tapping in the young, but not in the elderly group. This result indicates that in self-paced tapping, the bimanual advantage is absent in elderly. Electrophysiological results revealed an interaction between tapping condition and age group on low beta band (14-20 Hz) activity. Beta activity varied depending on the tapping condition in the elderly but not in the young group. Source estimations localized this effect within left superior parietal and left occipital areas. We interpret our results in terms of engagement of different mechanisms in the elderly depending on the tapping mode: a 'kinesthetic' mechanism for UM and a 'visual imagery' mechanism for BM tapping movement.

Keywords EEG - Oscillation · Parietal lobe · Tapping task $\cdot$ Aging $\cdot$ Sensory reafference

\section{Introduction}

Normal aging has been repeatedly associated with a decline in sensorimotor functions (Bugnariu and Fung 2007; Berard et al. 2009; Degardin et al. 2011). Age-related deficits in motor coordination are notably illustrated by evidence that the elderly exhibit temporal impairments even in basic sensorimotor tasks consisting of synchronized tapping with two hands at an isofrequency rate (Swinnen et al. 1998; Serrien et al. 2000; Wishart et al. 2000). Although the stability of synchronization between the two hands (i.e., the relative phase) during simultaneous (in-phase) movements of both indexes is not dependent on age, the stability of the relative phase decreases in the elderly during more complex anti-phase movements (alternate movements of both indexes; Serrien et al. 2000). Similarly, age-related increases in the variability of the inter-tap interval (ITI) 
have also been reported during simple repetitive unimanual (UM) and bimanual (BM) in-phase finger tapping (Sommervoll et al. 2011).

Converging lines of evidence suggest that the high motor instability during BM coordination in elderly might be the result of a deficit in intrinsic afferent information processing (Serrien et al. 2000). Firstly, an age-related weakening of movement-related proprioception has been reported during passive detection of motion or position occurring in the lower (Diener et al. 1984; Fitzpatrick and McCloskey 1994) or the upper limbs (Ferrell et al. 1992; Wright et al. 2011). Since kinesthetic reafferences constitute an important source of information on the produced movements, a deficit in integrating these reafferences should likely result in enhanced motor instability. Secondly, the elderly have been shown to benefit more than the young from visual feedback (Seidler-Dobrin and Stelmach 1998; Wishart et al. 2002; van Hedel and Dietz 2004), suggesting that with aging sensory reafferences compensate less reliably for a lack of other sources of feedback to stabilize motor coordination. Greater age-related differences in BM coordination have indeed been found in the performance of motor tasks based only on intrinsic information (vision and/or proprioception) than when augmented visual feedback was available (Swinnen et al. 1998). A third level of evidence supporting the fact that motor instability in the elderly might be the result of a decline in the processing of sensory reafferences comes from comparing UM and BM tapping tasks. ITIs have been shown to be less variable during synchronous BM than during UM tapping movement in young adults (Helmuth and Ivry 1996), an effect known as the 'bimanual advantage'. Because BM movements integrate twice as many effectors as UM movements, more kinesthetic reafferences are available in BM than in UM movement. This could account for the decrease in ITI variability in BM conditions (Helmuth and Ivry 1996). In addition, kinesthetic reafferences could modulate ITI variability by having an effect on the temporal coupling between the two effectors (Drewing and Aschersleben 2003), with the additional tactile and kinesthetic reafferences provided by the two hands helping to maintain the coupling stability. These assumptions are supported by the work of Drewing and Aschersleben (2003) who showed that right hand ITI variability increases when the left hand simultaneously taps into a gap (reduced tactile reafferences). Because the bimanual advantage depends on kinesthetic reafferences, the alteration of kinesthetic reafferences with aging would result in a reduction in bimanual advantage in the elderly compared to the young.

The neurophysiological consequences of aging on the processing of kinesthetic reafferences also remain unclear. Sensory brain regions such as primary sensorimotor cortices and the superior and posterior parietal cortices have been involved in tasks requiring the processing of kinesthetic reafferences, notably in BM (Serrien et al. 2001; Swinnen and Wenderoth 2004) and UM coordination tasks (Lacquaniti et al. 1995; Koeneke et al. 2004). Others sensory area such as the occipital lobe was also showed involved during motor imagery of a finger sequence opposition task (Guillot et al. 2009). With aging, additional activity during inter-limb coordination was found in the parietal cortex and in the cerebellum, two regions already known to be involved in high-order sensorimotor coordination during demanding tasks (Heuninckx et al. 2008). Brain oscillatory activity in the beta band has been pointed out as a mechanism supporting the maintenance of motor or cognitive states (Alegre et al. 2003; Engel and Fries 2010). In general, power modulations of brain oscillations relate to the overall level of activity in neuronal populations (see Kilavik et al. 2013). Decrease in power (desynchronization) corresponds to an activated brain state and is a correlate of excited cortical neurons (Steriade and Llinas 1988; Pfurtscheller et al. 1997; Kilavik et al. 2013). Conversely, an increase in power (synchronization) reflects decreased cortical excitability or increased inhibition of neuronal populations (Pfurtscheller et al. 1997; Kilavik et al. 2013). For example, the voluntary suppression of complex finger movements was associated with increased upper alpha power over the sensorimotor cortex compared to a rest condition (Sauseng et al. 2013). Thus, a power decrease or increase reflects changes in cerebral activation level. A decrease in beta oscillations during movement was related to the sensorimotor network (Pfurtscheller and Lopes da Silva 1999) suggesting that beta oscillations are linked to the association of sensory and motor aspects. A decrease in beta activity has been reported during finger movements (Gaetz et al. 2010) and the sequence of rhythmic movements (Alegre et al. 2003). Additionally, Keinrath and collaborators (2006) showed a modulation of beta oscillation in sensorimotor regions during active and passive movements as well as during kinesthetic illusions. These findings suggest that beta oscillations within sensorimotor regions during $\mathrm{UM}$ and $\mathrm{BM}$ tapping movements might reflect the processing of kinesthetic reafferences.

To investigate the role of the beta oscillations and to examine whether kinesthetic reafferences decline with age, we compared self-paced UM and BM tapping movements in young and elderly participants. We did not provide any visual or auditory feedback on tapping movements during the task in order to ensure that the performance relied purely on kinesthetic reafferences. To identify the neural basis for the age-related effects on motor coordination, electroencephalogram was recorded during the tapping tasks. 


\section{Materials and methods}

Participants

Twenty-nine young adults (14 males; aged $24 \pm 2$ years, mean \pm SD; range 19-29 years) and 27 elderly adults (12 males; aged $69 \pm 5$ years; range $60-83$ years) participated in the study. The elderly participants were both active and independent, community-dwelling individuals. All participants had normal or corrected-to-normal vision and were not on any medication, and none reported a history of major medical disorders, sustained head injury, psychiatric or neurological disorders, alcohol or drug abuse. They were all right-handed $(87 \pm 18$, mean $\pm \mathrm{SD}$. Edinburgh handedness inventory score: $100=$ max right-handed and $-100=$ max left handed) according to a twelve-item version of the Oldfield Edinburgh handedness inventory (Oldfield 1971). All participants gave written informed consent to take part in the study. The Ethics Committee for research on humans participants (University of Lausanne) and the Faculty of Psychology and Educational Science (University of Geneva) approved all experimental procedures.

\section{Methods}

Participants were comfortably seated in front of a computer screen at a distance of about $60 \mathrm{~cm}$. Two joysticks were placed inside two boxes used to attenuate auditory feedback from button-press and to avoid the possibility of visual feedback on hand movements. The joysticks were positioned $20 \mathrm{~cm}$ in front of each participant at a distance of $30 \mathrm{~cm}$ from each other. To prevent the participants from hearing the sounds from the joysticks' buttons, participants wore earplugs. Two loudspeakers delivered the tones of a metronome (low-pitched tone: $500 \mathrm{~Hz}$ ) and the imperative signal (high-pitched tone: $4000 \mathrm{~Hz}$ ) at an intensity of $70 \mathrm{dBSPL}$ at the location where the participants were sitting (measured using an audiometer Testo 816, Testo AG, Kirchzarten). We asked to each participant if they discriminated easily the tones of the metronome. All participants confirmed that the loudness was sufficient and comfortable. Stimuli delivery and response recording were monitored by Presentation 14.4 software (Neurobehavioral System, Albany, CA).

Participants performed tapping tasks in which they were instructed to press the joysticks' buttons with their index fingers. At the beginning of each block, the frequency of the auditory metronome was set at $2 \mathrm{~Hz}$ for $5 \mathrm{~s}$. Participants were instructed to produce symmetrical BM button presses on each beat of the metronome. After these $5 \mathrm{~s}$ of rhythmic auditory stimulation, the metronome was turned off and the participants had to continue self-pacing by pressing the buttons at the same tempo as when the metronome was active (BM condition). Following a randomly varying delay (see below), a high-pitched tone was presented, prompting the participant stop the left hand tapping while continuing right hand tapping at the same pace (UM condition). The next high-pitched tone prompted the participant to switch to BM tapping again, and so on. Each UM and BM trial lasted from 4 to 7 s (i.e., from 8 to 14 manual taps). Participants performed between 10 and 12 blocks of about $60 \mathrm{~s}$, consisting of 6 trials in the BM condition and 5 trials in the $\mathrm{UM}$ condition, for a total of $60 \mathrm{BM}$ trials and $50 \mathrm{UM}$ trials. The proper 10 experimental blocks were performed after 2 familiarization blocks. Participants could take short rest periods between blocks if they wished. The whole experiment lasted approximately $15 \mathrm{~min}$. EEG was recorded during the whole experimental session.

\section{Behavioral analyses}

Inter-tap intervals (ITI) between each button press of the right index were calculated. Mean (ITImean) and standard deviation (ITIsd) of inter-tap intervals were computed on correct responses for each of the UM and BM tapping conditions. ITImean indexes the rhythm of the produced tempo and ITIsd its variability. Steadiness of performance was tested by the variability of the ITI in series of 8-14 taps. Series with a minimum of 4 consecutives correct taps were included in the calculation of the variability. The first tap after the switching was excluded to guarantee the steadiness of the behavior. Symmetric tapping (i.e., BM movement) was considered to be correct if the delay between the button presses of each hand did not exceed $50 \mathrm{~ms}$. ITImean and ITIsd were separately submitted to a $2 \times 2$ mixed design ANOVA with age (Young; Elderly) as betweensubject factor and tapping condition (UM; BM) as withinsubject factor.

\section{Electrophysiological recordings}

Continuous EEG was recorded at a sampling rate of $2048 \mathrm{~Hz}$ through a 64-channel Biosemi ActiveTwo amplifier system (Biosemi, Amsterdam, Netherlands) referenced to the CMS-DRL ground which functions as a feedback loop driving the average potential across the montage as close as possible to amplifier zero. Eye blinks were recorded by two electrodes placed above and below the right eye, while horizontal eye movements were recorded by two supplementary electrodes placed on the exterior side of each eye. The final electrode positions were not measured in each individual subject, but a template cap was used.

Offline analyses of the EEG were performed using Cartool software (developed by D. Brunet, Functional Brain 
Mapping Laboratory, Geneva, Switzerland). Raw EEG data were first down-sampled to $1024 \mathrm{~Hz}$ to reduce computational load and band-pass filtered (0.34-40 Hz, Notch $50 \mathrm{~Hz}$ and DC removed). We used the median tap (4th tap) in a sequence of 7 consecutive taps (in UM or BM condition) to select epoch of interests. For each trial of each condition, the duration of epochs of interest was $500 \mathrm{~ms}$ (from $250 \mathrm{~ms}$ before to $250 \mathrm{~ms}$ after the median tap). Electrodes were visually defined as being artifacted and were then interpolated using a spherical spline interpolation (Perrin et al. 1987). On average, $5.4 \%( \pm 2.2$, range $0-6)$ of the 64 electrodes were interpolated. In addition to a $\pm 100 \mu \mathrm{V}$ peak to peak artifact rejection criterion, each epoch containing eye blinks or other noise transients were excluded. The EEG data were re-referenced to the mean voltage of all 64 channels (i.e., transformed to the common average reference). On average, the number of epochs included in the analysis was $54 \pm 6$ (rejection rate of $6.8 \%$ ) for the $\mathrm{BM}$ and of $50 \pm 5$ (rejection rate of $7.8 \%$ ) for the UM condition.

\section{EEG analyses}

The analyses of oscillatory activity were conducted according to previously published methods (e.g., see Arzy et al. 2010; Bernasconi et al. 2011). For each participant and condition, a fast Fourier transform approximation (FFTA; Lehmann and Michel 1990) was applied to each epoch and electrode in the following four frequency bands: low alpha $(\mathrm{A} 1=8-10 \mathrm{~Hz})$, high alpha $(\mathrm{A} 2=10-12 \mathrm{~Hz})$, low beta $(\mathrm{B} 1=14-20 \mathrm{~Hz})$, and high beta $(\mathrm{B} 2=20-30 \mathrm{~Hz})$ with a frequency resolution of $2 \mathrm{~Hz}$. We subdivided the alpha and beta frequencies band to increase the frequency space resolution and to dissociate the function of each sub-frequency. Previous evidence indeed indicates that different frequency bands within the alpha frequency range reflect different cognitive processes. Lower alpha $(7-9.5 \mathrm{~Hz})$ has been related to attentional processes and upper alpha $(9.5-12 \mathrm{~Hz})$ to sensory-semantic information (Klimesch et al. 1997; Neuper and Pfurtscheller 2001). In the same vein, beta rhythms have been observed in different beta sub-frequency bands according to the part of the body involved during voluntary movements. Thereby, the largest beta activity was observed in 16-21 Hz for finger movement and $19-26 \mathrm{~Hz}$ during foot movement (Neuper and Pfurtscheller 1996; Pfurtscheller et al. 1997). The outputs of this analysis (i.e., FFTA power maps) were averaged across conditions for each frequency band and participant. By comparison with classical FFT power representing squared potential values, the FFTA provides the FFT for each channel and then uses the complex values of all electrodes to calculate the first principal component for each frequency point. By contrast with the FFT power map, the FFTA preserves the polarity information and is reference-independent thereby allowing source estimations to be directly calculated in the frequency domain.

Of note, movement-related beta changes have been previously characterized by a decrease followed by an increase in power. Precisely, a premovement desynchronization phase (ERD) about $1 \mathrm{~s}$ before the movement followed with a post-movement synchronization phase (ERS or beta rebound) has been reported. The ERD phase has been advanced to correspond to the preparation and the execution of the movement, while the ERS phase has been associated either to sensory reafferences, to the return of the motor cortex to a 'rest' state or to the closure of the motor process (see Pfurtscheller et al. 1996; Derambure et al. 1999; Alegre et al. 2003, 2004). Because we have selected a 500-ms period, our analyses might have missed potential power modulations during this interval. However, the previously reported fast beta changes were mainly described during discrete hand/foot movements during time intervals of $8 \mathrm{~s}$ or more (Pfurtscheller et al. 1996; Derambure et al. 1999; Pfurtscheller and Lopes da Silva 1999; Pfurtscheller 2001; Alegre et al. 2003) but were not observed for each movement in a sequence of two consecutive movements (Alegre et al. 2004). Post-movement ERS was indeed not observed after the first movement but only at the end of the sequence of movements. In our study, we focused on a 500$\mathrm{ms}$ period in the middle of a long sequence of movement. Hence, based on previous literature, beta ERD could be expected to be stable around each tap of continuous tapping movement as in the current study.

In each frequency band, modulations of frequency power for all conditions and groups were assessed using the global power spectra (GPS) by averaging the absolute value of the frequency power of all electrodes. The main advantage of using the GPS is that it does not require the (arbitrary) selection of electrodes of interest, which could induce experimenter's biases, and allows having more data-driven analysis. GPS in each frequency band were then submitted to a $2 \times 2$ mixed design ANOVA with age (Young; Elderly) as between-subject factor and tapping condition (UM; BM) as within-subject factor. This first analysis served to determine the frequency bands in which significant modulation manifested. Source estimations were then calculated and statistically compared across conditions for the frequency bands showing significant GPS modulations.

The sources of oscillatory activity were estimated by applying a distributed linear inverse solution based on the local autoregressive average (LAURA; Grave de Peralta Menendez et al. 2001, 2004). LAURA selects the source configuration that better mimics the biophysical behavior of electric fields (i.e., activity at one point depends on the activity at neighboring points according to electromagnetic law). The accuracy of the estimation of sources from a 64-scalp electrode was shown sufficient 
to determine the deep source activity in the brain (Michel et al. 2004). The solution space was based on a realistic head model and included 4,996 nodes selected from a $3 \times 3 \times 3 \mathrm{~mm}$ grid equally distributed within the gray matter of the Montreal Neurological Institute's average brain. Several validation and clinical studies demonstrated the accuracy of distributed source localization, mostly by investigating epileptic patients (see Michel and Murray 2012 for review). We applied statistical parametric mapping analyses to the source estimation and thus even if the estimated activity in brain regions was of unrealistic size, statistical analysis revealed whether differences between experimental conditions were reliable. One ANOVA was conducted at each of the 4,996 solution points. Correction for multiple comparisons was implemented by considering as significant only the clusters of at least 14 contiguous nodes. This spatial criterion was determined using the AlphaSim program (available at http://afni.nimh.nih.gov). The 10,000 Monte Carlo permutations were performed using the 4,996 nodes of our lead field matrix and revealed a false-positive probability $<0.001$ for observing a cluster of 14 nodes. We interpreted only the most pronounced effects by choosing to focus on the maxima: solution point with the minimal $p$ value within each cluster. For the frequency bands of interest, source estimations were calculated for each participant and each condition and then submitted to $2 \times 2$, age group $\times$ tapping condition ANOVA.

The normality of the distributions of the behavioral and EEG data was assessed using a Kolmogorov-Smirnov test, which did not reach our $p<0.05$ significance criterion (all $p$ values $>0.2$ ) except in the alpha frequency band. Tukey HSD test was used as post hoc analyses when necessary.

\section{Results}

\section{Behavior}

Analyses of the mean inter-tap interval (ITImean) revealed no significant main effects or interaction of the factors age and tapping condition (all $p$ values $>0.05$; Table 1 ). Analyses of the inter-tap interval variability (ITIsd) revealed a tendency on the age factor with a larger rhythm variability

Table 1 Inter-tap interval (ITI) mean and variability in the bimanual and unimanual tapping condition for the young and the elderly groups

\begin{tabular}{lll}
\hline ITI mean \pm SD $(\mathrm{ms})$ & Bimanual & Unimanual \\
\hline Young & $484.30 \pm 12.98$ & $483.44 \pm 14.24$ \\
Elderly & $485.33 \pm 17.29$ & $483.90 \pm 16.54$ \\
\hline
\end{tabular}

in old than young participants $(F(1,54)=3.33, p=0.07)$ and a significant age $\times$ tapping condition interaction $(F(1,54)=4.94, p=0.03)$. Tukey HSD post hoc test revealed a greater variability of tapping rhythm in UM than $\mathrm{BM}$ in the young group only ( $p<0.05$; Fig. 1$)$.

\section{EEG}

Analyses of the oscillatory activity, as indexed by the global power spectra (GPS), revealed a main effect of tapping condition in both low and high beta bands (B1 and B2), with lower power in BM than UM in both age groups $(F(1,54)=6.18, p<0.01$ and $F(1,54)=1.81, p<0.01$, for $\mathrm{B} 1$ and B2, respectively). In B1 $(14-20 \mathrm{~Hz})$, there was a significant interaction between age and tapping condition $(F(1,54)=4.67, p<0.05)$, driven by a lower power in BM than UM in the elderly (Tukey HSD test, $p<0.05$; Fig. 2). We found no other significant GPS differences. We conducted multiple Pearson linear correlations between intertap variability (ITIsd) and frequency power values (GPS) in low and high beta band in each age group (young; old) and condition (UM; BM). No significant correlations were observed.

ANOVAs on the source estimations were computed from the FFTA topographies of the B2 and B1 band. For $\mathrm{B} 2$, there was a main effect of tapping condition in the right cingulate cortex (cingulate gyrus, limbic lobe. BA $24 ; F(1,54)=25.66, p<0.01)$, with a lower activity in BM than UM (Fig. 3a, right panel). For B1, there was a main effect of tapping condition within the right premotor cortex with a decrease in power during BM than UM (sub-gyral frontal lobe. BA 6; $F(1,54)=33.64, p<0.01$; Fig. 3a, left panel). There was also a significant age $\times$ tapping condition interaction for B1 within two clusters. The maxima of the first cluster were localized within the left occipital lobe (Middle occipital gyrus, BA 19) and showed a lower power in the elderly during BM than UM condition $(F(1,54)=12.28, p<0.01$; Fig. 3b, left panel $)$. The maxima of the second cluster were localized within the left superior parietal lobule (post-central gyrus, BA 5; $F(1,54)=11.47, p<0.01$; Fig. 3b, right panel $)$ and showed a lower power in the elderly during UM than BM condition $(p<0.05)$. Power tended to decreased during BM than $\mathrm{UM}(p=0.07)$ within the left superior parietal lobule in the young group (Fig. 3b, right panel).

\section{Discussion}

We investigated the effect of aging on the behavioral and electrophysiological responses during self-paced unimanual and bimanual tapping movements. Behaviorally, we found a bimanual advantage in the young, but not in the 
Fig. 1 Inter-tap interval variability in the bimanual and unimanual tapping condition for the young and the elderly groups $* p<0.05 ;{ }^{*} p=0.06$, HSD Tukey test

Fig. 2 Global power spectra across frequency bands for the young and the elderly groups in the bimanual (BM) and unimanual (UM) tapping condition. ${ }^{*} p<0.05 ; * * p<0.01$, HSD Tukey test
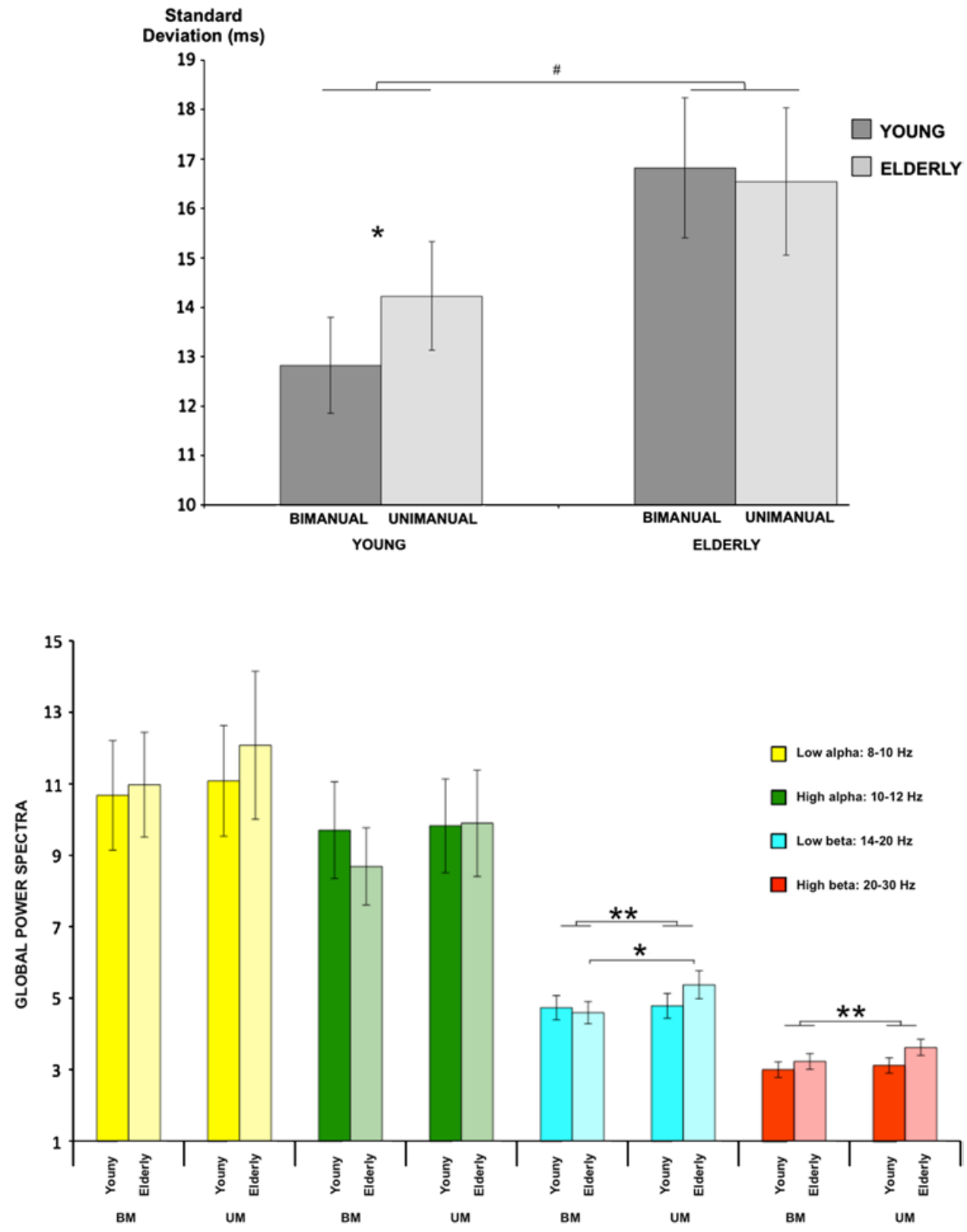

elderly. Electrophysiologically, in the elderly group, the EEG analyses showed a lower oscillatory activity in the 14$20 \mathrm{~Hz}$ beta band during bimanual compared to unimanual tapping movements. Source estimations revealed that this interaction followed from modulations in beta activity in the left occipital and left superior parietal areas. Since the experimental conditions were not compared to a baseline, the results were interpreted as a difference between the conditions and age group during the tapping task, independently on whether the global oscillatory activity changed compared to when participants were not tapping.

The behavioral results did not reveal any effect of age on the mean inter-tap intervals (ITI) and a marginally significant age-related increase in the ITI variability in both tapping conditions, although the result did not reach the conventional $0.05 p$-value level. Impacts of age on the variability of motor coordination, but not on its average accuracy, are typically reported in motor control studies (Galganski et al. 1993; Wishart et al. 2000; Bangert et al. 2010; Summers et al. 2010; Sommervoll et al. 2011). Our results do not show any increase in variability in the elderly during self-paced tapping.

In addition, we observed a decrease in the ITI variability during bimanual but not unimanual tapping in the young group. This bimanual advantage supports previous evidence showing a decrease in tapping variability when both hands are used (Helmuth and Ivry 1996; Drewing and Aschersleben 2003; Bangert et al. 2010). Furthermore, our 


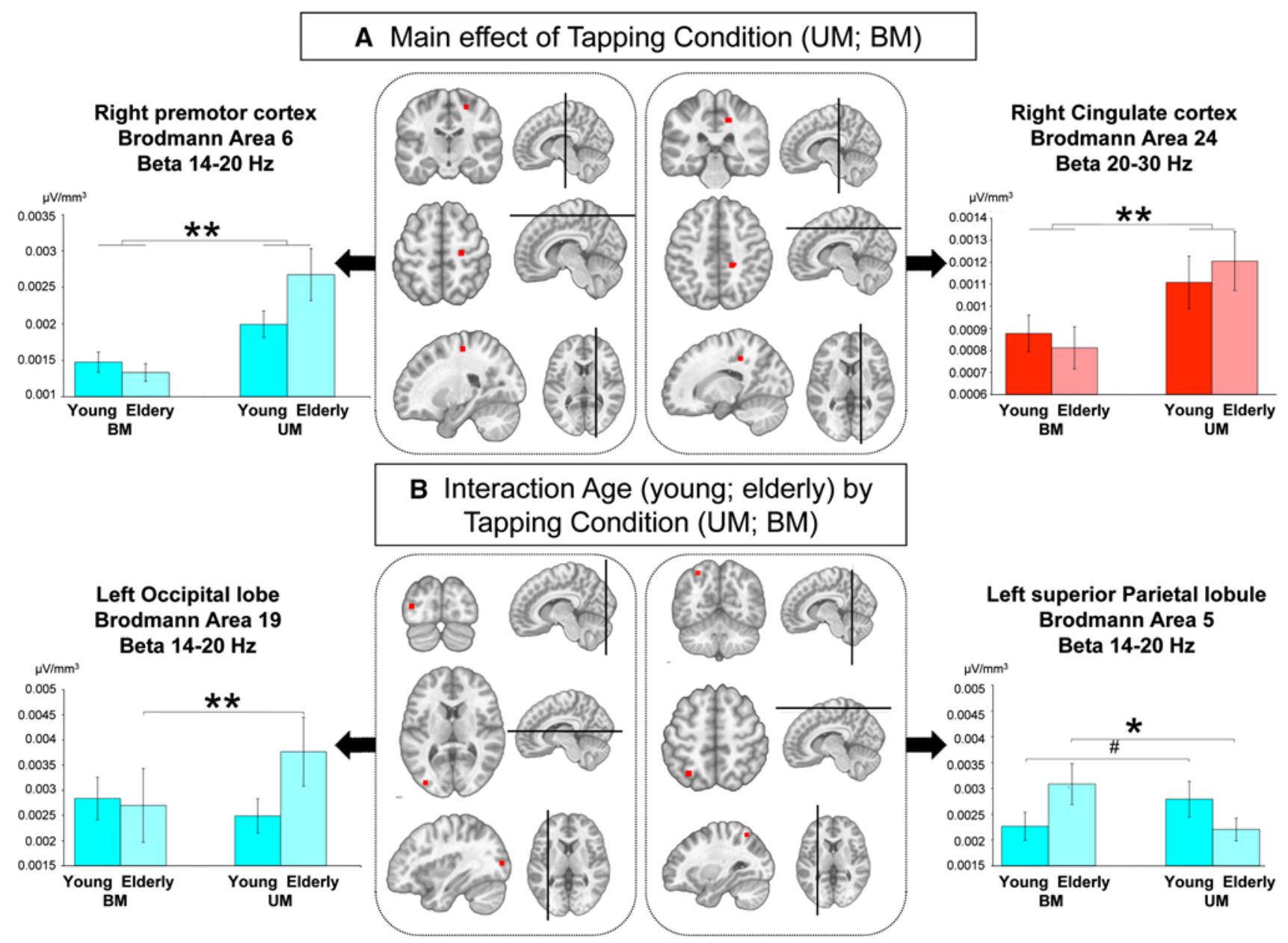

Fig. 3 a The two-by-two age $\times$ condition ANOVA on the electrical source estimations of the beta generators revealed a main effect of Tapping condition within the right premotor cortex (sub-gyral frontal lobe, BA $6 ; 14-20 \mathrm{~Hz}$ ) and the right cingulate cortex (cingulate gyrus, limbic lobe, BA $24 ; 20-30 \mathrm{~Hz}$ ). b There was a significant age $\times$ Tapping condition interaction in the left occipital lobe (mid-

results confirm the hypothesis of similar ITI variability in bimanual tapping as in unimanual tapping in the elderly group. We suppose that the absence of bimanual advantage in the elderly indicates a decline in sensory reafferences. During self-paced unimanual and bimanual tapping movements performed under sole kinesthetic reafference control (considered to be a more difficult setting for movement), the elderly did not benefit from the additional hand in inter-tapping stabilization that is observed in young adults (Helmuth and Ivry 1996; Drewing and Aschersleben 2003). Supporting this hypothesis, when visual feedback is present, bimanual advantage is maintained in the elderly (Bangert et al. 2010; see also Sasaki et al. 2011) providing evidence that guided-paced movements are easier to perform than self-paced movements. Two suppositions support the concept of bimanual advantage. The first involves separate timing mechanisms associated with dle occipital gyrus, BA $19 ; 14-20 \mathrm{~Hz}$ ) and the left superior parietal lobule (postcentral gyrus, BA 5; 14-20 Hz). Red points correspond to the minimal $p$ values. The bar graphs depict the mean activity of the beta generators at the locus of the minimal $p$ value. ${ }^{*} p<0.05$, $* * p<0.01,{ }^{\#} p=0.07$ HSD Tukey test

each effector. In bimanual movement, the outputs of these timing mechanisms are averaged (Helmuth and Ivry 1996; Drewing and Aschersleben 2003). As we consider that action timing is based on its sensory reafferences (Aschersleben and Prinz 1995) and that our experimental settings excluded any visual or auditory feedback, we hypothesize that the variability of the ITI was mainly dependent on kinesthetic reafferences. Our results support the second supposition assuming the role of sensory reafferences in temporal coupling (Drewing and Aschersleben 2003), in which bimanual movement is enhanced as a result of the second hand's additional kinesthetic reafferences (Aschersleben and Prinz 1995). Age-related bimanual tapping variability could therefore be interpreted as a consequence of inefficient processing of movement-related kinesthetic information. Supporting this hypothesis, it is known that tactile and proprioceptive information is impaired with the process 
of aging (Diener et al. 1984; Ferrell et al. 1992; Fitzpatrick and McCloskey 1994; Wright et al. 2011). Similarly, elderly subjects have been shown to experience more difficulties while performing cycling bimanual movements when they have to rely exclusively on their intrinsic sources of sensory information (Swinnen et al. 1998).

Our EEG results revealed a marginally significant decrease in beta power in the left superior parietal lobule (BA 5, posterior parietal cortex: PPC) in the young group during the bimanual as opposed to the unimanual tapping condition. This region is known to play a major role in the integration of sensorimotor information (Serrien et al. 2001; Wenderoth et al. 2005) and more specifically in kinesthetic processing (Debaere et al. 2003) while carrying out motor activity. This tendency for a decrease in beta activity in the parietal lobule supports the hypothesis that kinesthetic reafferences account for bimanual advantage.

In contrast and as a probable consequence of the agerelated decline of kinesthetic reafferences (Diener et al. 1984; Ferrell et al. 1992; Madhavan and Shields 2005; Shaffer and Harrison 2007; Goble et al. 2009; Wright et al. 2011), there was a significant increase in beta power in the left superior parietal lobule in the elderly when both hands were used as opposed to the right hand alone. This result suggests that the motor system does not favor a tactile kinesthetic mechanism in order to control bimanual tapping movements that in turn might explain the absence of reduced bimanual variability in the elderly. In addition, the kinesthetic mechanism seems to be involved only during unimanual tapping. In line with previous interpretations (Muller et al. 2003; Kilavik et al. 2013), we associate the decreases in beta power during movement production with the amount of sensorimotor processing rather than with purely motor processes.

Another hypothesis concerning the role ascribed to the PPC during sensorimotor tapping concerns its anticipatory motor control function (see Blakemore and Sirigu 2003; Krause et al. 2012). The principle is that the PPC compares the actual feedback-related response with the anticipated movement of the stimulus at each movement and sends the information about the error of matching to the cerebellum. Hence, the PPC would play a role in adjusting the internal model in order to improve the synchronization for the subsequent taps. Using unimanual synchronization tapping task (with visual and auditory externally cues), Krause and collaborators (2012) reported a major role of the left PPC for anticipatory motor control over its multisensory integration function. The design of our study which do not manipulate pacing modalities do not allow to interpret our data set in light of the anticipatory motor function of the PPC. Further research would be necessary to investigate the role of the PPC in anticipatory motor control with aging.
A last aspect to consider is the link between working memory and internal representation of rhythms. It was shown that the internal time representations rely on attentional and working memory systems primarily localized within prefrontal and parietal areas (e.g., Lewis and Miall 2003; Serrien and Brown 2004). In our study, these systems might have played a role since the participants had to maintain a previously learned beat during the tapping. Because working memory tends to decrease with aging (Craik and Byrd 1982), tapping performance across our group of interest might have been influenced by such unspecific factors. We would note, however, that tapping movement is a simple coordination task performed relatively automatically, and automatic timing mechanisms remain intact in normal aging (Wishart et al. 2000; Summers et al. 2010).

In the elderly, the lower beta power in the left occipital lobe (BA 19) during bimanual tapping signifies that this region is more activated when the two hands are engaged. Changes in power are evidently related to motor cortical activation/inhibition since power modulations in alpha and beta frequency bands are inversely correlated with the fMRI-BOLD signal during a bimanual motor task (Ritter et al. 2009). We postulate that this specific activation within the left occipital lobe in the older participants might rely on a visual imagery mechanism to control bimanual movements. This hypothesis is reinforced by several neuroimagery studies. Using MEG, recent research showed a decreased of alpha and beta power over occipital area during mental rotation task (van Wijk et al. 2013) and mental stimulation of movement (de Lange et al. 2008). In a motor imagery study, multiple occipital areas (BA 17, 18 and 19) have been shown activated during visual imagery of a finger sequence opposition task (Guillot et al. 2009). Interestingly, in the same study, the left and right superior parietal lobules (BA 5) were not activated during visual imagery, but they were during kinesthetic imagery of the same sequence. In addition, when older participants were required to perform the imagery task of stepping over an obstacle, the fMRI results emphasized an extensive network of cortical areas involved in visuomotor transformation, including visual association areas (BA 19), that were activated more in older than in younger participants (Wai et al. 2012). A recent study showed in a deafferented patient a decrease in the quality of sensory reafference and an increased in the quality of visual imagery during a mental rotation task (ter Horst et al. 2012). Moreover, a reduction in beta power has been shown during imagery of manual movement (McFarland et al. 2000; Nakagawa et al. 2011). Our observation supports the theory that older participants rely on visual imagery in order to compensate for the poor quality of kinesthetic reafferences when performing bimanual tapping. 
Age-related decline is characterized by two distinct mechanisms combining behavioral and neurophysiological observations. Both mechanisms being associated with an age-related increase in cerebral activation (i.e., brain oscillation power decrease): Compensation is accompanied by a similar behavioral performance level between age groups (Cabeza 2002; Mattay et al. 2002; Reuter-Lorenz and Lustig 2005; Heuninckx et al. 2008), whereas dedifferentiation is associated with a poorer performance level in older than younger subjects ( $\mathrm{Li}$ and Lindenberger 1999). In our study, the general decrease in GPS in low beta band during the BM condition compared to the UM condition, together with the absence of bimanual advantage in older subjects, would be compatible with a dedifferentiation mechanism. However, since no correlation was established between behavioral and electrophysiological data, this hypothesis cannot be formally confirmed.

Two other beta generators were identified as distinguishing two tapping conditions irrespective of age. The right premotor cortex (rPMC; BA 6; low beta band) and the right cingulate cortex (BA 24; high beta band) showed a decrease in beta activity during bimanual as opposed to unimanual tapping movements in both age groups. The involvement of areas in the right hemisphere could arise from the supplementary movement performed by the left hand during the bimanual condition compared to the unimanual condition. Previous studies on motor control have shown a left hemispheric dominance during bimanual movements and the preferential involvement of contralateral brain areas during unimanual movements (Jancke et al. 1998; Serrien et al. 2003; Serrien and Brown 2004; Serrien 2008). The lower right hemispheric activity during the right hand movement might follow from transcallosal inhibitory inputs from the left hemisphere (Beaule et al. 2012). In our study, the left hemisphere might be involved during both conditions, whereas the right hemisphere might be involved during bimanual condition only. The fact our finding corroborates well-established effects can be considered as a positive control that our methodological approach was appropriate and reliable for the investigation of our theoretical questions.

\section{Conclusions}

We have provided supplementary evidence on the agerelated changes in electrocortical oscillations during self-paced unimanual and bimanual tapping performed under tactile and proprioceptive reafferences condition. Electrical source estimations of oscillatory activity identified two main regions involved in age-related differences in beta oscillation during manual tapping. In the elderly, decreased beta oscillatory power was observed in the left superior parietal lobule during unimanual movement and in the left occipital lobe during bimanual movement. We interpret these results as reflecting the involvement of a kinesthetic and a visual imagery mechanism, respectively. The elderly would switch between these two mechanisms that control each tapping condition using a visuomotor imagery control during bimanual movements and a kinesthetic control during unimanual movements. In addition, the present data suggest that a decline in kinesthetic processing accounts for the increased variability of isofrequency tapping and in particular for the loss of bimanual advantage with aging.

Acknowledgments This work was supported by a grant from the Rhône-Alpes Region to ES, CL, MPD and JB (Research Cluster Fund No. 0801398101 ) and from the Swiss National Science Foundation to LS (\#320030_143348). Cartool software (http://sites.google.com/ site/fbmlab/cartool) has been programmed by Denis Brunet, from the Functional Brain Mapping Laboratory, Geneva, Switzerland, and supported by the Center for Biomedical Imaging (CIBM) of Geneva and Lausanne. Recordings were performed in the Laboratory for Experimental Research on Behavior (www.unil.ch/lerb) at the University of Lausanne, Switzerland, and at the Faculty of Psychology and Educational Sciences at the University of Geneva, Switzerland (www.u nige.ch/fapse). The authors wish to thank Fosco Bernarsconi for his assistance with the analyses and the volunteers for their participation.

\section{References}

Alegre M, Gurtubay IG, Labarga A, Iriarte J, Malanda A, Artieda J (2003) Alpha and beta oscillatory changes during stimulusinduced movement paradigms: effect of stimulus predictability. NeuroReport 14:381-385

Alegre M, de Gurtubay IG, Labarga A, Iriarte J, Malanda A, Artieda J (2004) Alpha and beta oscillatory activity during a sequence of two movements. Clin Neurophysiol 115:124-130

Arzy S, Allali G, Brunet D, Michel CM, Kaplan PW, Seeck M (2010) Antiepileptic drugs modify power of high EEG frequencies and their neural generators. Eur J Neurol 17:1308-1312

Aschersleben G, Prinz W (1995) Synchronizing actions with events: the role of sensory information. Percept Psychophys 57:305-317

Bangert AS, Reuter-Lorenz PA, Walsh CM, Schachter AB, Seidler RD (2010) Bimanual coordination and aging: neurobehavioral implications. Neuropsychologia 48:1165-1170

Beaule V, Tremblay S, Theoret H (2012) Interhemispheric control of unilateral movement. Neural Plast 2012:627816

Berard JR, Fung J, McFadyen BJ, Lamontagne A (2009) Aging affects the ability to use optic flow in the control of heading during locomotion. Exp Brain Res 194:183-190

Bernasconi F, Manuel AL, Murray MM, Spierer L (2011) Pre-stimulus beta oscillations within left posterior sylvian regions impact auditory temporal order judgment accuracy. Int J Psychophysiol 79:244-248

Blakemore SJ, Sirigu A (2003) Action prediction in the cerebellum and in the parietal lobe. Exp Brain Res 153:239-245

Bugnariu N, Fung J (2007) Aging and selective sensorimotor strategies in the regulation of upright balance. J Neuroeng Rehabil 4:19

Cabeza R (2002) Hemispheric asymmetry reduction in older adults: the HAROLD model. Psychol Aging 17:85-100

Craik FIM, Byrd M (1982) Aging and cognitive processes. Plenum Press, New York 
de Lange FP, Jensen O, Bauer M, Toni I (2008) Interactions between posterior gamma and frontal alpha/beta oscillations during imagined actions. Front Human Neurosci 2:7

Debaere F, Wenderoth N, Sunaert S, Van Hecke P, Swinnen SP (2003) Internal vs external generation of movements: differential neural pathways involved in bimanual coordination performed in the presence or absence of augmented visual feedback. NeuroImage 19:764-776

Degardin A, Devos D, Cassim F, Bourriez JL, Defebvre L, Derambure P, Devanne H (2011) Deficit of sensorimotor integration in normal aging. Neurosci Lett 498:208-212

Derambure P, Defebvre L, Bourriez JL, Cassim F, Guieu JD (1999) Event-related desynchronization and synchronization. Reactivity of electrocortical rhythms in relation to the planning and execution of voluntary movement. Neurophysiol Clin 29:53-70

Diener HC, Dichgans J, Guschlbauer B, Mau H (1984) The significance of proprioception on postural stabilization as assessed by ischemia. Brain Res 296:103-109

Drewing K, Aschersleben G (2003) Reduced timing variability during bimanual coupling: a role for sensory information. Q J Exp Psychol 56:329-350

Engel AK, Fries P (2010) Beta-band oscillations-signalling the status quo? Curr Opin Neurobiol 20:156-165

Ferrell WR, Crighton A, Sturrock RD (1992) Position sense at the proximal interphalangeal joint is distorted in patients with rheumatoid arthritis of finger joints. Exp Physiol 77:675-680

Fitzpatrick R, McCloskey DI (1994) Proprioceptive, visual and vestibular thresholds for the perception of sway during standing in humans. J Physiol 478(Pt 1):173-186

Gaetz W, Macdonald M, Cheyne D, Snead OC (2010) Neuromagnetic imaging of movement-related cortical oscillations in children and adults: age predicts post-movement beta rebound. NeuroImage 51:792-807

Galganski ME, Fuglevand AJ, Enoka RM (1993) Reduced control of motor output in a human hand muscle of elderly subjects during submaximal contractions. J Neurophysiol 69:2108-2115

Goble DJ, Coxon JP, Wenderoth N, Van Impe A, Swinnen SP (2009) Proprioceptive sensibility in the elderly: degeneration, functional consequences and plastic-adaptive processes. Neurosci Biobehav Rev 33:271-278

Grave de Peralta Menendez R, Gonzalez Andino S, Lantz G, Michel CM, Landis T (2001) Noninvasive localization of electromagnetic epileptic activity. I. Method descriptions and simulations. Brain Topogr 14:131-137

Grave de Peralta Menendez R, Murray MM, Michel CM, Martuzzi R, Gonzalez Andino SL (2004) Electrical neuroimaging based on biophysical constraints. NeuroImage 21:527-539

Guillot A, Collet C, Nguyen VA, Malouin F, Richards C, Doyon J (2009) Brain activity during visual versus kinesthetic imagery: an fMRI study. Hum Brain Mapp 30:2157-2172

Helmuth LL, Ivry RB (1996) When two hands are better than one: reduced timing variability during bimanual movements. J Exp Psychol Hum Percept Perform 22:278-293

Heuninckx S, Wenderoth N, Swinnen SP (2008) Systems neuroplasticity in the aging brain: recruiting additional neural resources for successful motor performance in elderly persons. J Neurosci 28:91-99

Jancke L, Peters M, Schlaug G, Posse S, Steinmetz H, Muller-Gartner $\mathrm{H}$ (1998) Differential magnetic resonance signal change in human sensorimotor cortex to finger movements of different rate of the dominant and subdominant hand. Cogn Brain Res 6:279-284

Keinrath C, Wriessnegger S, Muller-Putz GR, Pfurtscheller G (2006) Post-movement beta synchronization after kinesthetic illusion, active and passive movements. Int J Psychophysiol 62:321-327

Kilavik BE, Zaepffel M, Brovelli A, Mackay WA, Riehle A (2013) The ups and downs of beta oscillations in sensorimotor cortex. Exp Neurol 245:15-26
Klimesch W, Doppelmayr M, Schimke H, Ripper B (1997) Theta synchronization and alpha desynchronization in a memory task. Psychophysiology 34:169-176

Koeneke S, Lutz K, Wustenberg T, Jancke L (2004) Bimanual versus unimanual coordination: what makes the difference? NeuroImage 22:1336-1350

Krause V, Bashir S, Pollok B, Caipa A, Schnitzler A, Pascual-Leone A (2012) $1 \mathrm{~Hz}$ rTMS of the left posterior parietal cortex (PPC) modifies sensorimotor timing. Neuropsychologia 50:3729-3735

Lacquaniti F, Guigon E, Bianchi L, Ferraina S, Caminiti R (1995) Representing spatial information for limb movement: role of area 5 in the monkey. Cereb Cortex 5:391-409

Lehmann D, Michel CM (1990) Intracerebral dipole source localization for FFT power maps. Electroencephalogr Clin Neurophysiol 76:271-276

Lewis PA, Miall RC (2003) Distinct systems for automatic and cognitively controlled time measurement: evidence from neuroimaging. Curr Opin Neurobiol 13:250-255

Li SC, Lindenberger U (1999) Cross-level unification : a computational exploration of the link between deterioration of neurotransmitter systems and dedifferentiation of cognitive abilities in old age. In: Nilsson LGM, Markowitsch H (eds) Cognitive neuroscience of memory. Hogrefe \& Hube, Seattle, pp 103-146

Madhavan S, Shields RK (2005) Influence of age on dynamic position sense: evidence using a sequential movement task. Exp Brain Res 164:18-28

Mattay VS, Fera F, Tessitore A, Hariri AR, Das S, Callicott JH, Weinberger DR (2002) Neurophysiological correlates of age-related changes in human motor function. Neurology 58:630-635

McFarland DJ, Miner LA, Vaughan TM, Wolpaw JR (2000) Mu and beta rhythm topographies during motor imagery and actual movements. Brain Topogr 12:177-186

Michel CM, Murray MM (2012) Towards the utilization of EEG as a brain imaging tool. NeuroImage 61:371-385

Michel CM, Murray MM, Lantz G, Gonzalez S, Spinelli L, Grave de Peralta R (2004) EEG source imaging. Clin Neurophysiol 115:2195-2222

Muller GR, Neuper C, Rupp R, Keinrath C, Gerner HJ, Pfurtscheller G (2003) Event-related beta EEG changes during wrist movements induced by functional electrical stimulation of forearm muscles in man. Neurosci Lett 340:143-147

Nakagawa K, Aokage Y, Fukuri T, Kawahara Y, Hashizume A, Kurisu K, Yuge L (2011) Neuromagnetic beta oscillation changes during motor imagery and motor execution of skilled movements. NeuroReport 22:217-222

Neuper C, Pfurtscheller G (1996) Post-movement synchronization of beta rhythms in the EEG over the cortical foot area in man. Neurosci Lett 216:17-20

Neuper C, Pfurtscheller G (2001) Event-related dynamics of cortical rhythms: frequency-specific features and functional correlates. Int J Psychophysiol 43:41-58

Oldfield RC (1971) The assessment and analysis of handedness: the Edinburgh inventory. Neuropsychologia 9:97-113

Perrin F, Pernier J, Bertrand O, Giard MH, Echallier JF (1987) Mapping of scalp potentials by surface spline interpolation. Electroencephalogr Clin Neurophysiol 66:75-81

Pfurtscheller G (2001) Functional brain imaging based on ERD/ERS. Vision Res 41:1257-1260

Pfurtscheller G, Lopes da Silva FH (1999) Event-related EEG/MEG synchronization and desynchronization: basic principles. Clin Neurophysiol 110:1842-1857

Pfurtscheller G, Stancak A Jr, Neuper C (1996) Event-related synchronization (ERS) in the alpha band-an electrophysiological correlate of cortical idling: a review. Int J Psychophysiol 24:39-46

Pfurtscheller G, Neuper C, Andrew C, Edlinger G (1997) Foot and hand area mu rhythms. Int J Psychophysiol 26:121-135 
Reuter-Lorenz PA, Lustig C (2005) Brain aging: reorganizing discoveries about the aging mind. Curr Opin Neurobiol 15:245-251

Ritter P, Moosmann M, Villringer A (2009) Rolandic alpha and beta EEG rhythms' strengths are inversely related to fMRI-BOLD signal in primary somatosensory and motor cortex. Hum Brain Mapp 30:1168-1187

Sasaki H, Masumoto J, Inui N (2011) Effects of aging on control of timing and force of finger tapping. Mot Control 15:175-186

Sauseng P, Gerloff C, Hummel FC (2013) Two brakes are better than one: the neural bases of inhibitory control of motor memory traces. NeuroImage 65:52-58

Seidler-Dobrin RD, Stelmach GE (1998) Persistence in visual feedback control by the elderly. Exp Brain Res 119:467-474

Serrien DJ (2008) The neural dynamics of timed motor tasks: evidence from a synchronization-continuation paradigm. Eur J Neurosci 27:1553-1560

Serrien DJ, Brown P (2004) Changes in functional coupling patterns during bimanual task performance. NeuroReport 15:1387-1390

Serrien DJ, Swinnen SP, Stelmach GE (2000) Age-related deterioration of coordinated interlimb behavior. J Gerontol 55:295-303

Serrien DJ, Nirkko AC, Lovblad KO, Wiesendanger M (2001) Damage to the parietal lobe impairs bimanual coordination. NeuroReport 12:2721-2724

Serrien DJ, Cassidy MJ, Brown P (2003) The importance of the dominant hemisphere in the organization of bimanual movements. Hum Brain Mapp 18:296-305

Shaffer SW, Harrison AL (2007) Aging of the somatosensory system: a translational perspective. Phys Ther 87:193-207

Sommervoll Y, Ettema G, Vereijken B (2011) Effects of age, task, and frequency on variability of finger tapping. Percept Mot Skills 113:647-661

Steriade M, Llinas RR (1988) The functional states of the thalamus and the associated neuronal interplay. Physiol Rev 68:649-742

Summers JJ, Lewis J, Fujiyama H (2010) Aging effects on event and emergent timing in bimanual coordination. Hum Mov Sci 29:820-830
Swinnen SP, Wenderoth N (2004) Two hands, one brain: cognitive neuroscience of bimanual skill. Trends Cogn Sci 8:18-25

Swinnen SP, Verschueren SMP, Bogaerts H, Dounskaia N, Lee TD, Stelmach GE, Serrien DJ (1998) Age-related deficits in motor learning and differences in feedback processing during the production of a bimanual coordination pattern. Cogn Neuropsychol 15:439-466

ter Horst AC, Cole J, van Lier R, Steenbergen B (2012) The effect of chronic deafferentation on mental imagery: a case study. PLoS ONE 7:e42742

van Hedel HJ, Dietz V (2004) The influence of age on learning a locomotor task. Clin Neurophysiol 115:2134-2143

van Wijk BC, Litvak V, Friston KJ, Daffertshofer A (2013) Nonlinear coupling between occipital and motor cortex during motor imagery: a dynamic causal modeling study. NeuroImage 71:104-113

Wai YY, Wang JJ, Weng YH et al (2012) Cortical involvement in a gait-related imagery task: comparison between Parkinson's disease and normal aging. Parkinsonism Relat Disord 18:537-542

Wenderoth N, Debaere F, Sunaert S, Swinnen SP (2005) The role of anterior cingulate cortex and precuneus in the coordination of motor behaviour. Eur J Neurosci 22:235-246

Wishart LR, Lee TD, Murdoch JE, Hodges NJ (2000) Effects of aging on automatic and effortful processes in bimanual coordination. J Gerontol 55:85-94

Wishart LR, Lee TD, Cunningham SJ, Murdoch JE (2002) Agerelated differences and the role of augmented visual feedback in learning a bimanual coordination pattern. Acta Psychol 110:247-263

Wright ML, Adamo DE, Brown SH (2011) Age-related declines in the detection of passive wrist movement. Neurosci Lett 500:108-112 